\title{
¿QUÉ HACE A UNA DECISIÓN BUENA? CRITERIOS DE TOMA DE DECISIONES DESDE EL MANAGEMENT CIBERNÉTICO
}

\section{WHAT MAKES A GOOD DECISION? CRITERIA TO THE MAKING DECISIONS FORM THE CYBERNETIC MANAGEMENT}

\author{
Gonzalo Flores-Castro Lingán \\ Universidad Católica San Pablo, Arequipa, Perú
}

\begin{abstract}
Resumen
El presente artículo intenta analizar la buena toma de decisiones bajo el paradigma del management cibernético, valiéndonos de la teoría de la acción de Juan Antonio Pérez López. Se exponen, en la introducción, algunos problemas sobre los criterios para considerar buena una decisión. Se desarrolla el moldeo perezlopiano para analizar todos los posibles resultados de una acción y encontrar los criterios de decisión a tomar en cuenta en una buena decisión. Al final se introducen las variables éticas implícitas en el modelo, que permiten tener una mirada holística de la buena decisión.
\end{abstract}

Palabras clave: Acción, toma de decisiones, ética, management, Juan Antonio Pérez López.

\footnotetext{
Abstract

This article attempts to analyze what constitutes good decision-making under the cybernetics management paradigm, using the action theory ofJuan Antonio Pérez López. Some problems with the criteria for identifying a decision as good are presented in the introduction. Perez López's theory was developed in order to analyze all possible results of human action and describe the decision criteria to be considered in determining whether a decision is good. In the conclusion, the ethical variables implicit in the model are introduced, which allow a holistic view of good decision-making.
} 
Key words: Action, decision-making, ethics, management, Juan Antonio Pérez López.

\section{Introducción}

En marzo 2001 Neal Paterson, consejero delegado de la Cerner Corporation, observó que el estacionamiento de la compañía tenía cada vez menos autos estacionados en horarios de oficina. Ante la sospecha que sus colaboradores trabajaban menos de las cuarenta horas establecidas, envió un e-mail a sus directivos en los siguientes términos: «Un gran número de nuestros empleados está trabajando menos de 40 horas. Como directivos, o no saben o no les importa lo que están haciendo sus colaboradores. En cualquier caso, hay un problema que o resuelven o los reemplazaré. Son responsables de lo que pasa. Tienen dos semanas». Los directivos de Cerner consiguieron que todo el mundo estuviera trabajando desde las ocho de la mañana hasta las cinco de la tarde. Lo que no anticipó Neal Patterson es que el autoritario e-mail fuese filtrado y colgado en Internet. El mensaje fue leído por analistas e inversores que quedaron sorprendidos por el lenguaje usado. El resultado: el precio de la acción de la compañía bajo un $22 \%$ en tres días. Toda la prensa económica de EE. UU. se hizo eco de este hecho, de modo que al negativo impacto económico hubo que añadirle la vergüenza personal para el consejero delegado (Ariño, 2005).

Esta decisión parece que estuvo mal por varias razones. Entre las cuales podemos encontrar razones económicas (la baja en el precio la acción), así como de imagen empresarial (prensa económica) e incluso de ambiente laboral (maltrato, uso injusto de poder, "traición" de un directivo, etc.). Sin embargo, de haber obtenido mayores beneficios económicos, por ejemplo, un incremento del $2 \%$ en las acciones, ¿se habría considerado la acción de Paterson como buena? En términos económicos la respuesta quizás sea afirmativa. De hecho, en su famoso artículo del New York Times Magazine en 1970, titulado «The social responsibility of business is to increase its profits», Friedman defendía la tesis de que las empresas no deben tener otra responsabilidad que la de maximizar sus beneficios. En otros términos, independientemente de las otras funciones que cumpla una empresa, el criterio máximo de actuación empresarial es la maximización de beneficios económicos y a ella debe de subordinarse la toma de decisiones empresariales. Así, una buena decisión empresarial es aquella que genere mayores beneficios económicos ${ }^{1}$.

Sin embargo, intuitivamente sabemos que esto no es así. El criterio económico no puede ser el único criterio para que una decisión sea calificada de buena. De hecho, los efectos de considerar la maximización de beneficios como criterio dominante los hemos sufrido en las crisis de la última década (Argandoña, 2010). Existen, pues, otras razones por las cuales consideramos una decisión como buena, pero resulta difícil explicitarlas. Como dice Higgins, «todos nos vemos motivados a tomar buenas decisiones... pero ¿qué hace que una decisión sea buena?» (Higgins, 
2000, p. 1218), es decir, ¿qué criterios tenemos para calificar a una decisión como buena? El asunto es peliagudo pues, para empezar, resulta difícil la definición del término "bueno". ¿A qué nos referimos con que una decisión es buena? ¿a que obtuvo beneficios económicos? ¿a que fue una decisión ética? ¿a que los involucrados salieron satisfechos emocionalmente? Los escolásticos dirían que "bueno" es un término análogo, por lo que se puede decir de muchas maneras (Feser, 2014), lo cual no nos dice mucho, porque bueno puede ser un resultado económico, así como puede existir una bondad ética.

No es el único problema. Por ejemplo, Melé (2009), quien toma a los escolásticos como base de su análisis (Melé, 2014), explica que existen tres tipos de bienes: aquellos llamados bienes útiles (por ejemplo, dinero), placenteros (por ejemplo, buen ambiente laboral) y humanos o morales (aquellos que nos perfeccionan como humanos, como la confianza en las personas o la buena reputación). Pues bien, ¿estos bienes están separados?, ¿es posible hablar de una decisión buena en un aspecto (útil) y mala en otro (moral)?, y si es así, ¿sería una decisión globalmente buena o mala? Aquí entramos en el problema de la integración de la bondad ética en la toma de decisiones (Argandoña, 2007; Flores Castro, 2018). La mayoría de las teorías dentro de la dirección de empresas no toma en cuenta aspectos éticos en sus decisiones (Torres, 2001; Rosanas, 2007a), tienen diversos problemas en sus supuestos teóricos y filosóficos (Ghoshal, 2005; Rosanas, 2009; Alcázar, 2010) en especial los antropológicos y éticos (Mitroff, 2004; Melé \& González, 2015).

Por otra parte, tenemos problemas epistemológicos para encontrar qué cosa hace a una decisión buena. Por ejemplo, abordar el asunto desde una perspectiva post hoc, haciendo revista a todas y cada una de las decisiones que fueron exitosas y obteniendo de ellas los principios por los cuales son exitosas, es un absurdo metodológico. Hacerlo así presupone los criterios por los cuales se califica a una decisión de "buena": escoger entre buenas y malas decisiones en una muestra presupone un criterio por el cual afirmamos "estas decisiones son buenas y por tanto podemos obtener criterios de decisión de ellas"; al escoger las buenas para saber por qué son buenas ya hemos usado un criterio para decir "son buenas", cayendo en una petición de principio.

Además, abordar el problema desde la perspectiva post hoc, es acercarse a la cuestión de manera descriptiva. Esto es problemático para la ciencia del management que, en general, está preocupada con resolver problemas prácticos, es decir, está preocupada de aquellas verdades útiles, aquellas que le permitan resolver sus problemas específicos (Rosanas, 2012)2. La lógica científica toma en cuenta proposiciones del tipo «Si Ax entonces By», en donde Ax explica causalmente a By y permite predecir su evento. Por ejemplo, si se bajan las tasas de interés, habrá más consumo; si se suben los precios de los productos habrá menos clientes, etc. Tendríamos que fabricar un "archivo» de todas las afirmaciones verdaderas «Si 
Ax entonces By» que sean consideradas buenas. Esto, desde el punto de vista práctico o de la acción, no tiene sentido, resulta poco práctico (e inaplicable en ciertas circunstancias) para cualquier directivo profesional (Pérez López, 1991). Es, en cualquier caso, preferible conseguir una serie de criterios formales, que prescindan del contenido real de la decisión, para poder ser usados como criterios universales de decisión.

Por todo esto, el presente artículo pretende exponer, de manera lógica, los criterios a priori que debe tener en cuenta un decisor para asegurar, al menos formalmente, que su decisión es una buena decisión. Para ello, nos basaremos en la teoría de la acción de Juan Antonio Pérez López (1991, 2014), que es relativamente poco conocida (Argandoña, 2007). Su trabajo es lo que él llama una lógica de la acción, que se enmarca en el paradigma del management cibernetics
(Melé, Chinchilla, \& López-Jurado, 2018). Además, el modelo de Pérez López integra diversos tipos de resultados de la acción, incluyendo los resultados éticos, de manera que puede considerarse una abstracción completa, es decir, un tipo de teoría que permite evaluar una acción de manera global: si puede ser considerada una buena decisión en todos los aspectos y no solo en uno (Rosanas, 2007b). Nuestro objetivo es exponer el moldeo mínimo para considerar a una decisión como buena, así como brindar razones suficientes para considerar los criterios que aquí expondremos. Así mismo, utilizaremos datos de diferentes ciencias para ir construyendo el modelo.

\section{Evaluación de una buena decisión}

El esquema mínimo para conceptualizar la interacción (acción-reacción) entre dos personas puede graficarse de la siguiente manera:

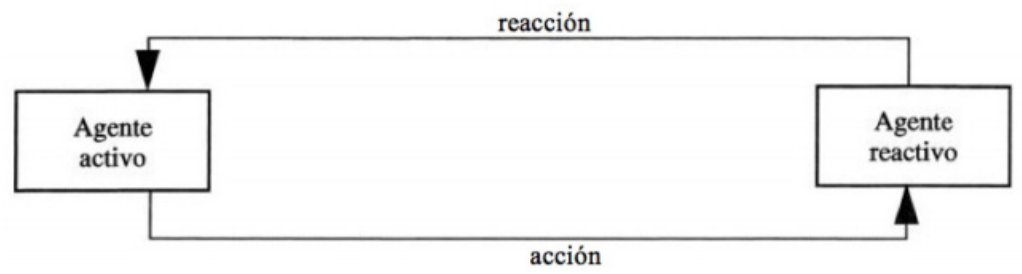

Figura 1. Modelo básico de sistema.

Fuente: Original de Teoría de la acción en las organizaciones: la acción personal de Pérez López (1991, p. 25)

Cuando el agente activo tiene un problema, es decir, se percata que «existe una situación que no es del todo agradable para una persona [el mismo u otra], o cuando se sospecha que existen situaciones más agradables que las actuales» (Ariño, 2005, p. 19), y que no puede solucionar solo; ejecuta una acción con otro agente (reactivo), con la esperanza que la acción de dicho agente (la reacción) solucione su problema. Esta acción se basa en una decisión 
previa y que, si soluciona su problema - es eficaz- puede ser considerada como una buena decisión. De hecho, la respuesta tradicional que se ha dado a la pregunta sobre si una decisión es una buena decisión es en términos de resultados, «en pocas palabras, una buena decisión es aquella que produce resultados positivos» (Higgins, 200o). Ahora bien, ¿qué se debe tomar en cuenta para conseguir esos "resultados positivos"? ¿qué criterios, evaluaciones o condiciones se deben tomar en cuenta?

Si realizamos un rápido análisis de esta interacción caemos en cuenta que tenemos, al menos, tres evaluaciones que debemos realizar para tratar de conocer $a$ priori la eficacia de la decisión del agente activo. En primer lugar, se debe analizar la proposición, o plan de acción, la díada «acción-reacción»; esto es, evaluar la validez de la proposición, que no es otra cosa que «la medida en que la acción y la reacción realmente resuelven el problema que se pretendía resolver» (Ariño, 2005, p. 30). En segundo lugar, debemos evaluar si el estado interno del agente activo es tal que le permite ejecutar el plan de acción; es decir, si le es factible u operativo ejecutar el plan de acción. Por último, hemos de evaluar si el estado interno del agente reactivo está en condiciones de realizar la reacción; o sea, si es operativo o instrumental (operatividad del agente reactivo) para dicho agente ejecutar su acción (la reacción).

En el ejemplo de Paterson, el problema parece haber sido que "las personas no están cumpliendo su horario de trabajo". En este caso, el plan de acción de Paterson era válido, porque la acción tomada, enviar el e-mail, ha resultado en la reacción por parte de los directivos, dar una orden a los subordinados para que estén cumplan cabalmente un horario de trabajo. Fue operativo, porque Paterson tenía el poder necesario para dar ese tipo de orden —un ultimátum-. Y fue instrumental porque los directivos subordinados a Paterson tenían también el poder necesario de dar la orden a sus empleados para que cumplan un determinado horario de oficina. Diferente hubiese sido el caso de otro directivo que sea inferior en la cadena de mando, pues no hubiese sido operativo para él pedirles a los otros directivos del mismo nivel utilizando un e-amil con un ultimátum.

Hasta aquí, podemos ver que fue una buena decisión. Esta evaluación de una acción concreta es lo que podemos llamar una evaluación estática. Sin embargo, en las organizaciones se requieren múltiples interacciones entre diversos agentes en distintos momentos, sea entre directivos y colaboradores, colaboradores con otros colaboradores, vendedores con clientes, etc. ¿Qué pasaría si los estados internos de los agentes cambian debido a las interacciones pasadas? La pregunta es clave para realizar un análisis completo. De hecho, la experiencia muestra que ese cambio existe y que influye en las futuras interacciones, incluso a nivel neuronal (Sapolsky, 2017). No tomar en cuenta el cambio de los estados internos de los agentes para la evaluación de una acción sería una abstracción incompleta. En 
ese sentido, al cambio producido en el agente activo luego de una interacción le llamaremos eficiencia - operatividad futura-, y al cambio producido en el agente reactivo le llamaremos consistencia -instrumentalidad futura-.

Ahora bien, el cambio al que nos referimos no es cualquier tipo de cambio, sino aquel «cambio que ocurra en los agentes como consecuencia de la ejecución del proceso de interacción, siempre que dicho cambio sea significativo para la explicación de las futuras interacciones» (Pérez, López, 1991). Para distinguirlo, utilizaremos el término aprendizaje, que es mucho más preciso. Dicho aprendizaje puede ser positivo, es decir, permite que las interacciones puedan continuar produciéndose en el futuro (o incluso facilitar las interacciones futuras); o negativo, esto es, un aprendizaje que dificulte o incluso imposibilite las futuras interacciones. Así, tenemos la evaluación mínima y completa de una interacción: un análisis estático - eficacia del plan de accióny un análisis dinámico -eficiencia y consistencia de un plan de acción-.

En el caso propuesto, parece ser que Paterson sólo realizó una evaluación estática de su decisión. Pues era eficaz (válida, operativa e instrumental), pero generó diversos aprendizajes en los agentes reactivos. Recibir un ultimátum no es algo que sea del todo agradable para algunos, y el no pensar en lo que podría haberles sucedido a sus directivos le pasó factura. De hecho, podría decirse que la decisión fue buena en su momento (resolvió el problema de los horarios, fue eficaz), pero la forma en que se hizo puso en riesgo la eficacia futura de la organización. Dicha decisión fue inconsistente, no se pensó en el aprendizaje de los directivos y ocasionó otro problema futuro. Pérez López (1992a) lo ejemplificaba con la fábula del pastorcillo mentiroso: un pastorcillo tenía un problema, estaba aburrido, y opto por un plan de acción para divertirse, iba a la taberna y gritaba "lobo, lobo"; viendo cómo los otros pastores corrían en respuesta a su falso pedido de auxilio, él se reía y divertía mucho; repitió esta fórmula dos o tres veces, y a la siguiente - cuando en verdad los lobos llegaronlos pastores ya no le creyeron y simplemente no fueron. Podría decirse que su forma de divertirse, aunque eficaz, era profundamente inconsistente, y por tanto una mala decisión, igual que la de Paterson.

\section{Evaluación dinámica:algunos ejemplos}

Cuenta Esopo que un día, un granjero, descubrió que su ganso había puesto un huevo de oro. Aunque escéptico al principio, pudo comprobar que era auténtico. El granjero estaba feliz de la buena suerte que había tenido, más aún cuando se repitió la extraña experiencia. Todos los días veía como su ganso ponía otro huevo de oro, llegando a ser muy rico. Pero, junto con su creciente fortuna crecieron la impaciencia y la codicia. Incapaz de esperar, el granjero decidió matar al ganso para obtener todo el oro de una vez. Pero al abrir el ave, la encontró vacía. Allí no había huevos de oro, y ya no había modo 
de conseguir ninguno más. El granjero había matado al ganso que los producía.

¿Fue una buena decisión la del granjero? Sencillamente no, pero ¿por qué? Porque no solo no fue eficaz (el ganso carecía de la instrumentalidad necesaria para dar los huevos de oro ante el corte fatal del granjero); sino, sobre todo, porque fue inconsistente. Dicha decisión afectó a aquello que podía solucionarle sus problemas económicos no solo ahora, sino también a futuro. De hecho, el granjero podría haber intentado reproducir al ganso, o realizar un tipo de análisis veterinario externo para verificar que hubiese huevos de oro, o realizar una operación quirúrgica experta sin dañar al ganso. Todas estas opciones podrían haber sido eficaces (o no), pero hubiesen respetado la eficacia futura (la consistencia). El pobre granjero pronto se encontró sin los medios que producen los huevos. Ahora bien, ¿qué pasaría si fuesen personas? Es el caso de Paterson, que obtuvo los huevos de oro (las personas estaban en su lugar de trabajo) a costa de la vida del ganso (el malestar de los directivos). Algo parecido puede pasar en otros tipos de contextos, pero peor.

Por ejemplo, un operario trabaja en una empresa que está creciendo rápidamente. Ve una oportunidad magnífica de ascenso dentro de la organización (problema). Ella está a cargo de una máquina y está ansiosa por causar una buena impresión en sus superiores. Produce en niveles óptimos: ningún tiempo muerto, nada de mantenimiento. La máquina trabaja día y noche, la producción es extraordinaria, los costos bajan, etc. (huevos de oro, eficacia)
Al cabo de un tiempo, dicha persona obtiene su ascenso. Pero, el sucesor del puesto hereda una máquina deteriorada que empieza a fallar. Tiene que realizar mantenimiento, los costos se disparan y la utilidad cae en picado. ¿A quién se culpará por la pérdida de la empresa? Al sucesor. Su predecesor destruyó la máquina, pero ni se ha enterado que ha sido su culpa. No se dará cuenta de lo mala que fue la decisión de sobre exigir la máquina sin tomar en cuenta que es lo que le pasaría a futuro, es decir, sin tomar el criterio de consistencia en la decisión’3.

De hecho, el ejemplo anterior oculta el criterio de eficiencia. La primera persona que usó la máquina y que ascendió, aprendió algo: que puede obtener un resultados —un ascenso- usando una máquina hasta el extremo. El problema es que, al fallar el reemplazo, no se enterará de que fue por su mala decisión; o, dicho de otra manera, no se enterará de que de que su decisión fue ineficiente. Esto es una de las peores cosas que le puede pasar a una persona, que su decisión sea eficaz e inconsistente, pero que no sepa que es inconsistente, pues es el peligro de un aprendizaje negativo, algo que la persona pensaba que era una buena decisión en el fondo era una decisión mala (Pérez López, 1991). Al menos Paterson se enteró gracias a la mala fama de la prensa norteamericana.

Hasta aquí tenemos los criterios claves para la buena toma de decisiones: debe de ser eficaz, eficiente y consistente. Eficaz porque una buena decisión debe resolver un problema; eficiente, porque 
una buena decisión busca que el decisor esté en mejores condiciones para poder resolver mejor los problemas futuros (un aprendizaje positivo en el agente activo); y consistente, porque una buena decisión aspira a que, a futuro, las personas con las que se interactúa estén en una mejor disposición para resolver los problemas del agente activo. Si a un futuro no se puede resolver el problema debido a la decisión, fue una mala decisión, especialmente en un contexto organizacional en el que las decisiones suelen repetirse constantemente con los mismos agentes. Como dice Pérez López, «si la decisión es de un solo uso, piénselo dos veces antes de usarla» (Pérez López, 1992b).

\section{Resultados y motivos de la acción:}

Teniendo en cuenta la Figura 1 y el análisis realizado, podemos afirmar que por cada decisión se pueden esperar tres tipos de consecuencias o resultados de la acción (Pérez López, 2014): resultados extrínsecos, o la misma acción del agente activo y la reacción del agente reactivo (lo que ambos hacen, la propia interacción) ${ }^{4}$; resultados intrínsecos, el aprendizaje del agente activo fruto de la decisión tomada; y resultados trascendentes, o el aprendizaje del agente reactivo fruto de la interacción. Estos resultados son «irreducibles entre sí» (Alcázar, 2010, p. 105), además de ser a posteriori, mientras que la evaluación del plan de acción por parte del agente activo es $a$ priori.

Distinguir entre ambos, resultados y criterios deevaluación, resulta útil para el análisis pues una cosa es la eficacia, eficiencia y consistencia a priori y otra a posteriori. Una decisión puede ser una buena decisión $a$ priori y no a posteriori. Y, como dijimos, una buena decisión es aquella que produce resultados positivos, es decir, a posteriori. He allí una dificultad teórica para saber qué es una buena decisión. Porque, como hemos visto, una decisión puede ser eficaz (resultado extrínseco positivo), pero haber tenido aprendizajes negativos por parte de ambos agentes, como el caso del pastorcillo: losagentes reactivos cada vez estaban menos dispuestos a colaborar con él (inconsistencia) y el pastorcillo no se enteró de que su decisión fue la que causó el deterioro de la "máquina" - comoen el casodel operario(ineficiencia). Hemos de afirmar que, entre ambas, lo fundamental es que la decisión sea buena a priori (a nivel de criterios), pues que la decisión sea buena a posteriori (a nivel de resultados) puede no estar bajo control del agente activo. Esto debido a las circunstancias que no siempre son la mismas (no se cumple el criterio ceteris paribus en la vida real), y porque el agente reactivo es libre, su reacción depende de su querer más que de la acción del agente activo 5 . Por otra parte, evaluar que una decisión sea buena a posteriori puede llevarnos a los problemas epistemológicos quevimos en la introducción.

Una buena decisión, por tanto, no es aquella que produzca resultados positivos a posteriori en todos los aspectos necesariamente - pues existe el factor suerte (Ariño \& Maella, 2009)—. Una buena decisión es aquella en la que se evalúa $a$ priori todos los posibles resultados de la acción, eligiendo los positivos en todos los aspectos. Al hacerlo pueden ocurrir dos 
cosas: tener suerte, no solo solucionando el problema, sino siendo consistente; en ese caso, la decisión es una buena decisión y habrá aprendido positivamente, sabrá que debe hacer e interiorizará los criterios de decisión —es eficiente, sabe por qué fue una buena decisión-; o no tener suerte, la acción fue ineficaz a posteriori, en este caso, al menos no caerá en aprendizaje negativo, sabrá que no debe realizar algún plan de acción, pero al menos habrá considerado todos los posibles resultados - no fue inconsistente o, al menos, la inconsistencia de la acción no se debió al agente activo de manera intencionada-.

Por otra parte, la consideración de los resultados de la acción nos lleva a la valoración de estos para la decisión. En otras palabras, «el logro de cualquiera de aquellos tres tipos de resultados, o de todos simultáneamente, puede llegar a ser motivo de las decisiones de una persona, es decir, puede ser un logro intentado en sus decisiones ${ }^{6}$ (Pérez, López, 2014, p. 55). Este sencillo esquema lógico nos muestra que los agentes pueden tener distintos motivos para la acción. Al respecto, Pérez López (2014, p. 55) afirma que «[los] aprendizajes pueden tener un gran valor para el agente activo... [No siendo] nada extraño que la consecución de dichos aprendizajes pueda ser un objetivo explícitamente buscado por las decisiones de una persona», pero añade inmediatamente que «también puede ocurrir que ambos aprendizajes no solo no sean buscados, sino que sean, por el contrario, ignorados a la hora de tomar las decisiones» (Pérez López, 2014, p. 55). De hecho, nada nos impide pensarque, en toda lógica, el agente activo tenga que actuar por todos los motivos al mismo tiempo; sin embargo, «en una acción intervienen ordinariamente los tres motivos, aunque con mayor intensidad unos que otros» (Ferreiro \& Alcázar, 2012, p. 65)

Tenemos por tanto tres motivos de la acción para agente: motivos extrínsecos o aquellos que buscan los resultados extrínsecos, es decir, el logro de satisfacciones - la resolución del problema que producen las interacciones-; motivos intrínsecos o la valoración de los resultados intrínsecos, a saber, la búsqueda del propio aprendizaje del agente activo; y los motivos trascendentes oaquellos que buscan resultados trascendentes, o sea, el aprendizaje del agente reactivo ${ }^{7}$. Cabe notar que, al realizar una acción, el agente activo puede tener un motivo ignorando otros, pero los resultados se dan con independencia de las intenciones del decisor. Por otra parte, no necesariamente se debe preferir un resultado a otro: si bien se deben tener en cuenta todos los resultados de la acción para que sea una buena decisión, no necesariamente debe de motivarnos uno más queotro. En otras palabras, la condición de una buena toma de decisiones es esta: se deben tomar decisiones eficaces, eficientes y consistentes sea por el motivo que sea.

\section{Relación estructural, relación operativa: tipos de aprendizaje y ética}

Dado el análisis anterior, podemos deducir que se encuentran dos tipos de relaciones entre los agentes, una subyacente y necesaria para la otra. Por una parte, podríamos hablar de las condiciones de posibilidad de la interacción (acción-reacción): el estado interno de los agentes 
- operacionalidad e instrumentalidaddebe ser tal (debe haber un mínimo necesario) que permita la interacción; de no ser así, no hay interacción posible. A esta relación subyacente le llamaremos relación estructural, esto es, «los grados de confianza que puedan existir entre ambas personas» (Pérez López, 2014, p. 156). Supuesto esta relación estructural (entre otras cosas, un mínimo de confianza), la relación toma otro cariz, el de la relación operativa o aquellas acciones y reacciones que de hecho solucionan los problemas del agente activo y del reactivo. Nótese que tanto la relación estructural como la relación operativa son solo abstracciones de la relación real (u organización real). Si la organización, la relación real, desea seguir en el tiempo, se ha de tomar decisiones tomando en cuenta los criterios de eficiencia y consistencia, es decir, velando por que los estados internos de los agentes tomen en cuenta los resultados intrínsecos y trascendentes de las acciones (como condición de posibilidad de cualquier interacción actual y futura), y que mejoren en la calidad de sus interacciones, a saber, que las acciones y reacciones solucionen cada vez mejor los problemas.

Ahora bien, dado que los motivos de la acción pueden lógicamente distintos, pueden existir al menos dos tipos de aprendizaje. Si me mueven a actuar los motivos extrínsecos estoy buscando mejorar la relación operativa (¿qué tipo de acción generará la reacción que busco?, ¿cómo puedo mejorar la eficacia de mis acciones para generar mejores reacciones?), tendré un resultado intrínseco (buscado o no) que es un aprendizaje operativo, "adquisición de un conocimiento o habilidad "técnica": aprender un idioma, manejar un auto, dominar una técnica financiera... etc.» (Ferreiro \& Alcázar, 2012, p. 61). Por otra parte, si me mueve a actuar motivos trascendentes estoy buscando la mejora en la relación estructural (¿el otro querrá seguir colaborando conmigo a futuro?, ¿lo que estoy haciendo está fortaleciendo nuestra relación, ¿le estoy tomando en cuenta?, ¿le ayudo a tomarme en cuenta en su decisión?), tendré un resultado intrínseco (querido o no) que es un aprendizaje estructural, se aprende «a tomar conciencia del impacto de... [la] acción sobre otras personas» (Ferreiro \& Alcázar, 2012, p. 62).

Esta distinción entre aprendizajes operativos y estructurales nos abre la puerta al criterio ético en la toma de decisiones. En primer lugar, tomar decisiones consistentes implica tener un mínimo de motivos trascendentes en nuestra toma de decisiones (aunque no sea el motivo principal). Esto quiere decir que estoy pensando en el posible resultado trascendente, ¿qué le pasará al otro cuando ejecute este plan de acción? Como no lo sabemos con toda certeza, podemos realizar una simulación: si el agente reactivo pusiera en práctica conmigo el mismo plan de acción que yo pretendo poner en práctica con él y con las mismas intenciones que me mueven a mí, ¿querría colaborar con él? (Ariño, 2005). Esta formulación no es otra que «la regla de oro», el conocido principio ético de valor universal ${ }^{8}$, por lo que puede afirmarse que la evaluación incluye una valoración ética en sus supuestos. 
Pero el asunto va más allá. Valorar lo que le sucederá a otra persona en la interacción, es decir, tener motivos trascendentes - sobre todo trascendentes-, es precisamente no utilizarlo simplemente como un medio sin, a su vez, también como un fin en sí mismo. La fórmula de conducta que acaba de expresarse no es otra que la del segundo imperativo categórico de Kant, y «es el principio [moral] más importante para regir las relaciones de la persona en la empresa como comunidad de persona que es» (Llano, 1997, p. 98). Este principio, también llamado «principio personalista» (citado por Wojtila, 2013, p. 41), puede ser formulado de manera positiva de la siguiente manera: «las personas deben ser tratadas no solo con respeto, sino con benevolencia y cuidado» (Melé, 2009, p. 81). Lo cual confirma la integración de la bondad ética en los criterios decisionales que estamos analizando.

Debe tomarse en cuanta que tanto «(a) prendizaje operativo y estructural son los dos tipos de resultados internos que se producen en la persona por el solo hecho de haber actuado, con independencia de lo que venga del exterior o de lo que les ocurra a las personas con las que interactúa» (Ferreiro \& Alcázar, 2012, p. 62); además, ambos pueden ser tanto positivos como negativos. La noción de aprendizaje nos permite introducir otro rasgo de la buena toma de decisiones ética de tradición aristotélica: la noción de virtud. Es el camino que redescubrió Alasdair MacIntyre (2010) en su proyecto
«Tras la Virtud» y que Pérez López reconoce explícitamente: «Hablar de ética sin mencionar las virtudes morales es como hablar de mecánica sin mencionar la gravitación: se estaría haciendo un discurso más o menos poético, pero nada que pueda parecerse a un análisis riguroso» (Pérez López, 1998, p. 28).

\section{Conclusiones}

Una decisión no puede considerarse buena solamente por haber conseguido resultados económicos. Siendo "bueno" un término que tiene muchos sentidos, podemos concluir que una buena decisión es aquella que toma en cuenta, a priori, todos los resultados posibles de la acción, es decir, es una que considera los criterios de eficacia, eficiencia y consistencia. Si una decisión es buena en todos esos sentidos, podemos decir que es una buena decisión, con independencia del resultado a posteriori de la decisión. La valoración moral o bondad ética de la acción está íntimamente integrada en estos criterios de toma de decisiones graciasa la noción deaprendizaje operativo y estructural contenidos en los criterios deeficienciay consistencia. Se toma en cuenta desde la regla de oro universal, pasando por el segundo imperativo kantiano o principio personalista, hasta llegar a la noción de virtud aristotélica, dependiendo del tipo de motivos por los que se tome una decisión. Por tanto, los criterios vistos hasta ahora pueden servir al decisor para valorar sus decisiones de tal manera que sepa, al menos formalmente, que su decisión es una buena decisión. 


\section{Referencias}

Alcázar, M. (2010). Las decisiones directivas: Una aproximación antropológica al logro de efiacia y de aprendizajes positivios en las organizaciones. (Tesis Doctoral). Universidad de Navarra, Pamplona, España.

Ariño, M. A. (2005). Toma de decisiones y gobierno de organizaciones. Barcelona: Deusto.

Ariño, M. Á., \& Maella, P. (2009). Iceberg a la vista. zera Edición. Barcelona: Empresa Activa.

Argandoña, A. (2006). Milton Friedman (1912-2006). CaixaBank Chair of Corporate Social Responsibility, OP-07-9.

Argandoña, A. (2007). Integrating Ethics into action theory and organizational theory. Journal of Business Ethics, 78, 435-446.

Argandoña, A. (2010). La dimensión ética de la crisis financiera. Mediterráneo Económico, 18, 183-198.

Covey, S. R. (1993). The seven habits of highly effective people. USA: Simon \& Schuster.

Ferreiro, P., \& Alcázar, M. (2012). Gobierno de personas en la empresa. 6ta. Edición. Lima: Planeta.

Feser, E. (2014). Scholastic Metaphysics. Germany: Editiones Schlasticae.

Flores-Castro, G. (2018). Integración de la ética en la toma de decisiones. Ilustro, 9, 53-80.

Fuster, J. M. (2016). Cerebro y libertad. Los cimientos cerebrales de nuestra capacidad para elegir. Barcelona: Ariel.

Ghoshal, S. (2005). Bad management theories are destroying good management practices. Academy of Management Learning E Education, 4(1), 75-91.

Higgins, E. T. (2000). Making a good decision: Value from fit. American Psychologist, 55(11), 1217-1230. 
Llano, C. (1997). Dilemas éticos de la empresa contemporánea. México D.F.: Fondo de Cultura Económica.

MacIntyre, A. (2010). After virtue. zera. Edición. Indiana: University of Notre Dame Press.

Melé, D. (2009). Business Ethics in Action. Seeking Human Excelence in Organizations. Londres: Palgrave Macmillan.

Melé, D. (2014). Re-thinking capitalism: What we can learn from Scholasticism. Journal of Business Ethics, 133(2), 293-304.

Melé, D., Chinchilla, M. N., \& López-Jurado, M. (2018). The "Freely Adaptive System". Application of this Cybernetic Model to an Organization Formed by Two Dynamic Human Systems. Philosophy of Management. doi: 10.1007 / s40926-018-0098-x

Melé, D., \& González, C. (2015). Fundamentos antropológicos de la dirección de empresas. Pamplona: EUNSA - Astrolabio.

Mitroff, I. I. (2004). An open letter to the Deans and Faculties of American Business Schools. Journal of Business Ethics, 54, 185-189.

Pérez López, J. A. (1991). Teoría de la acción humana en las organizaciones. La acción personal. Madrid, España: Rialp.

Pérez López, J. A. (1992a). Apuntes sobre la teoría de la acción humana en las organizaciones. Bogotá: Universidad de la Sabana.

Pérez López, J. A. (1992b). Cómo generar confianza. Buenos Aires, Argentina. Obtenido de: https://www.youtube.com/watch?v=VRTM3XsVGyo

Pérez López, J. A. (1998). Liderazgo y ética en la dirección de empresas. Bilbao: Deusto.

Pérez López, J. A. (2014). Fundamentos de Dirección de Empresas. 6ta. Edición. Madrid: Rialp.

Rosanas, J. M. (2007a). Beyond economic criteria: A huministic approach to organizational survival. IESE Business School, 654, 1-22.

Rosanas, J. M. (2007b). On the methodology of Management Research. IESE Business School, 692. doi: http://dx.doi.org/10.2139/ssrn.1026241 
Rosanas, J. M. (2009). Pero, ¿Qué es el management? Revista de Antiguos Alumnos del IEEM, 12(1), 31-36.

Rosanas, J. M. (2012). Methodology and research in Management. International Journal of Management and Economics, 36, 8-19.

Sapolsky, R. (2017). Behave. The biology of humans at our best and worst. New York: Penguin Press.

Torres, M. B. (2001). Character and Decision-Making. (Tesis Doctoral). Universidad de Navarra, Pamplona, España.

Wojtyla, K. (2013). Amor y Responsabilidad. Madrid: Palabra. 


\section{Notas}

1 En honor al famoso economista, añadimos lo siguiente: «La tesis de Friedman (...) no es arbitraria ni, como se ha dicho en ocasiones, una muestra de capitalismo salvaje. Es una consecuencia lógica de los supuestos que sustentan su modelo. Friedman llevó a cabo un formidable trabajo explotando las consecuencias de esos supuestos, con lógica e inventiva...» (Argandoña, 2006, p. 7).

2 Nótese que esto no es un utilitarismo epistemológico. No afirmamos que el criterio de verdad sea la utilidad, es decir "es verdadero porque es útil"; afirmamos algo distinto, "de lo verdadero, lo útil". Es decir, se usan abstracciones de realidades con ciertos fines utilitarios; pero siguen partiendo de la realidad y se ajusta a ella, tomando todas las variables relevantes al problema (Rosanas, 2012).

3 Los ejemplos los tomo de Covey (1993).

4 Nótese que la operacionalidad e instrumentalidad actuales no son resultado de la interacción, sino sus condiciones de posibilidad.

5 La libertad es un supuesto de todo el sistema de Pérez López (Alcázar, 2010), el cual cuenta con sólidos fundamentos filosóficos (Mele, 2011, 2014) y científicos (Fuster, 2016). Según Melé, Chinchilla y López-Jurado (2018), «Pérez López did not debate the so-called problem of free will (is free will real or a mere illusion?), but his implicit assumption-like Aristotle-is that free will is not an illusion and we are not fully determined by external or biological factors». El tema es discutible, pero afirmamos junto con Fuster que «...es precisamente en el crisol de probabilidades e incertidumbres del cerebro humano donde cobra vida la libertad. La capacidad para escoger entre posibilidades proviene literalmente de la varianza y los grados de libertad de innumerables variables que subyacen a la acción humana futura. Como pasa con la evolución, el determinismo y la causalidad directa se disuelven en la probabilidad y, al hacerlo, ceden ante un factor teleológico: la finalidad, el objetivo» (Fuster, 2016, p. 17).

6 Motivo es la realidad en cuanto se prevé que resolverá el problema del agente, siendo por tanto valorada. En ese sentido, motivo y valor son lo mismo (Ferreiro \& Alcázar, 2012).

7 La búsqueda del aprendizaje del agente activo (resultado intrínseco) es un motivo intrínseco y produce el resultado intrínseco, mientras que la búsqueda del resultado trascendente es un motivo trascendente que produce un resultado intrínseco no buscado directamente (un aprendizaje en el agente activo que le 
facilita la consideración de resultados trascendentes), no produce el resultado trascendente (esto depende también del agente reactivo).

8 Se encuentra en distintas tradiciones sapienciales y religiosas, aunque con algunas variaciones. Estas tradiciones incluyen al Antiguo Egipto, el Zoroastrismo, la Filosofía Griega (Pítaco, Diógenes, Sexto Empírico, Isócrates y Epicteto), Judaísmo, Cristianismo, Islamismo, Sufismo, Hinduismo, Jansenismo, Sijismo, Confucianismo, Taoísmo, Budismo, Shintoismo, Humanismo Secular, Espiritualidad de los Nativos Americanos, Proverbios Africanos, entre otros (Melé, 2009).

Recibido: 11 de noviembre de 2019

Aceptado: 20 de diciembre de 2019 